\title{
A função do cardiologista para prevenção da Miocardiopatia Diabética: uma revisão narrativa
}

The role of the cardiologist for the prevention of diabetic cardiomyopathy: a narrative review

El papel del cardiólogo en la prevención de la miocardiopatía diabética: una revisión narrativa

Diego Miléo de Oliveira Freitas ${ }^{1 \star}$, Armando Hiroyuki Mori Júnior ${ }^{1}$, Poliana Campos Melli².

\section{RESUMO}

Objetivo: Revisar e definir sobre a Miocardiopatia Diabética como doença importante no escopo da insuficiência cardíaca com fração de ejeção preservada e a função do cardiologista em sua prevenção. Revisão bibliográfica: O Diabetes Mellitus é uma doença crônica e não transmissível, etiologicamente multifatorial, que altera o sistema cardiovascular ocasionando a Miocardiopatia Diabética, relacionada tanto aos diabéticos tipo I quanto ao tipo II, cuja fisiopatologia é complexa, onde diversas vias intracelulares alteradas e lesões celulares crônicas, resultantes do meio extracelular hiperglicêmico, geram alterações estruturais e funcionais do miocárdio, culminando em insuficiência cardíaca crônica, muitas vezes refratária aos tratamentos atuais. Assim, é fundamental que o médico cardiologista tenha a visão de iniciar a pesquisa precoce desta patologia desde o início do diagnóstico do diabetes, pois o coração pode apresentar alterações sutis, diagnosticadas apenas em exames de imagem, além de conscientizar estes pacientes a adotarem estilos de vida mais saudáveis e focar no controle glicêmico. Considerações finais: Levando em consideração a gravidade da doença, são necessários mais estudos prospectivos com foco em terapias específicas e melhor cuidado no manejo destes pacientes.

Palavras-chave: Complicações do diabetes, Insuficiência cardíaca, Cardiologia.

\begin{abstract}
Objective: Review and define Diabetic Cardiomyopathy as an important disease in the scope of heart failure with preserved ejection fraction and cardiologist's role in its prevention. Bibliographic review: Diabetes Mellitus is a chronic and non-transmissible disease, etiologically multifactorial, which alters the cardiovascular system causing Diabetic Cardiomyopathy, related to both type I and type II diabetics, whose pathophysiology is complex, where several intracellular pathways are altered and chronic cells injuries, resulting from the hyperglycemic extracellular environment, generate structural and functional changes in the myocardium, causing chronic heart failure, often refractory to current treatments. In this way, it is essential that the cardiologist has the vision to start the early research of this pathology from the beginning of the diagnosis of diabetes, because the heart can show subtle changes, diagnosed only in imaging exams, in addition to making these patients aware to adopt styles of healthier lives and focus on glycemic control. Final considerations: Taking into account the severity of the disease, further prospective studies are needed with a focus on specific therapies and better care in the management of these patients.
\end{abstract}

Keywords: Diabetes complication, Heart failure, Cardiology.

\footnotetext{
${ }^{1}$ Hospital Santa Júlia, Manaus - AM. *E-mail: d-m-o-f@hotmail.com

2 Hospital Samel, Manaus - AM.
} 


\section{RESUMEN}

Objetivo: Revisar e definir sobre la Miocardiopatía Diabética como una enfermedad importante en el ámbito de la insuficiencia cardíaca con fracción de eyección conservada y el papel del cardiólogo en su prevención. Revisión bibliográfica: La Diabetes Mellitus es una enfermedad crónica y no transmisible, etiológicamente multifactorial, que altera el sistema cardiovascular provocando Miocardiopatía Diabética, relacionada tanto con los diabéticos tipo I como con el tipo II, cuya fisiopatología es compleja, donde se alteran varias vías intracelulares y lesiones crónicas. Las células, que resultan del entorno extracelular hiperglucémico, generan cambios estructurales y funcionales en el miocardio, que culminan en insuficiencia cardíaca crónica, a menudo refractaria a los tratamientos actuales. Por ello, es fundamental que el cardiólogo tenga la visión para iniciar la investigación temprana de esta patología desde el inicio del diagnóstico de diabetes, ya que el corazón puede presentar cambios sutiles, diagnosticados solo en exámenes de imagen, además de concienciar a estos pacientes de adoptar estilos de vida más saludables y centrarse en el control glucémico. Consideraciones finales: Teniendo en cuenta la gravedad de la enfermedad, se necesitan más estudios prospectivos con un enfoque en terapias específicas y una mejor atención en el manejo de estos pacientes.

Palabras clave: Complicaciones de la diabetes, Insuficiencia cardíaca, Cardiología.

\section{INTRODUÇÃO}

O Diabetes Mellitus (DM) é uma doença crônica, não transmissível, caracterizada poliúria, polidipsia e polifagia, classificada conforme a International Diabetes Federation (IDF) em Diabetes Mellitus de Tipo 1 (DM1), Diabetes Mellitus de Tipo 2 (DM2), Diabetes Mellitus Gestacional (DMG) e outros tipos menos comuns. Foi descrita inicialmente em 1550 a.C. pelos egípsios através do papiro de Ebers e Aretaeus da Capadócia (século II d.C.) foi o primeiro médico a descrever a patologia. Edouard Laguesse, em 1893, identificou as ilhotas pancreáticas como secretoras de uma substância responsável pela redução da glicose sanguínea e, em 1921, Frederick Banting e Charles Best, reverteram a doença em animais, utilizando células betapancreática saudáveis, comprovando a relação glicemia-insulina (ROMANCIUC M, 2017; CASTANHOLA ME, et al., 2020; COSTA BB e MOREIRA TA, 2021).

No ano de 2015, gerou $14,5 \%$ de mortalidade global na faixa etária entre 20 e 79 anos de idade e um gasto de $11,6 \%$ das despesas totais de saúde, com aumento previsto para 10,4\% em 2040. Estatísticas globais inferem que 382 milhões de pessoas possuem DM, e esse número poderá chegar a 592 milhões em 2035 e 628,6 milhões em 2040, sendo DM2 responsável por $85 \%$ dos casos, dessa forma configurando um grave problema de saúde pública mundial por estar associada a distúrbios vasculares que geram disfunções em diversos órgãos como retina, rins, coração e nervos, sendo fator de risco independente para insuficiência cardíaca (ROMANCIUC M, 2017; KUL S, et al., 2018; CASTANHOLA ME, et al., 2020; COSTA BB e MOREIRA TA, 2021; FIRMINO SM, 2021).

A relação do Diabetes Mellitus é conhecida desde o século passado, quando, Lundbaek K (1969) observou disfunção miocárdica em idosos portadores da doença, porém o termo miocardiopatia diabética, consagrouse quando Rubler S, et al. (1972), durante estudos de necrópsias, constatou insuficiência cardíaca em diabéticos sem alterações das artérias coronárias ou hipertensão. No ano de 1974, Hamby et al., demonstrou a presença de nódulos de Kimmelstein-Wilson, patognomônicos de diabetes, associados à hipertrofia e fibrose miocárdicas (RAMIREZ R, et al., 2017; SALDARRIAGA-GIRALDO C, et al., 2019; PAVÓN-ROJAS AJ, et al., 2020).

A doença induz nos cardiomiócitos alterações estruturais e funcionais, como aumento do metabolismo de ácidos graxos, reduz oxidação de glicose, conduzindo à produção ineficiente de energia e transtornos no acoplamento excitação-contração, contribuindo para disfunção microvascular, remodelamento da matriz extracelular, fibrose, esteatose que somadas, irão gerar insuficiência cardíaca sistólica e diastólica (RAMIREZ R, et al., 2017; SALDARRIAGA-GIRALDO C, et al., 2019; PAVÓN-ROJAS AJ, et al., 2020).

A miocardiopatia diabética é caracterizada pela Insuficiência Cardíaca (IC) diastólica, quando ocorre alteração no relaxamento ou pressão de enchimento do ventrículo esquerdo, conhecida como Insuficiência 
Cardíaca com Fração de Ejeção Preservada (ICFEP), muitas vezes assintomática, ocorrendo em 27 a 69\% dos diabéticos, decorrente da hiperglicemia que leva à disfunção microvascular coronariana, causando isquemia subendocárdica, sendo o melhor exame para diagnóstico o ecodopplercardiograma (COELHO RCG, 2018; SOCIEDADE BRASILEIRA DE CARDIOLOGIA (SBC), 2018; SALDARRIAGA-GIRALDO C, et al., 2019; WEBER TR, et al., 2020; FIRMINO SM, 2021).

Aproximadamente $12 \%$ dos diabéticos têm insuficiência cardíaca e nos mais idosos pode chegar a $22 \%$, sendo o tratamento baseado no controle dos fatores de risco, mudanças no estilo de vida e uso de diuréticos para melhorar os sintomas congestivos (COELHO RCG, 2018; SBC, 2018; SALDARRIAGA-GIRALDO C, et al., 2019; WEBER TR, et al., 2020; FIRMINO SM, 2021).

Dessa forma, o presente estudo visou revisar na literatura a cerda desta patologia e alertar, principalmente os médicos cardiologistas, da necessidade de investigar sinais de insuficiência cardíaca em seus pacientes, mesmo nos assintomáticos, com objetivo de iniciar precocemente, medidas eficazes para interromper a progressão da doença.

\section{REVISÃO BIBLIOGRÁFICA}

\section{Epidemiologia da Insuficiência Cardíaca}

O médico cardiologista é o especialista nas doenças cardiovasculares, orientando na sua prevenção e estabelecendo tratamentos medicamentosos e até cirúrgicos, quando necessário. Conhecer o conceito de miocardiopatia diabética por esses profissionais é fundamental, pois o diabético tem elevada chance apresentar lesões em artérias e musculatura cardíacas pelos elevados índices glicêmicos, sejam eles a médio ou longo prazo. Invariavelmente, pacientes portadores de diabetes apresentam outras comorbidades como hipertensão arterial, obesidade, doença vascular periférica, doença renal e sinais de insuficiência cardíaca que estão no escopo de estudo deste profissional (SOUSA MM, et al., 2017; WEJNER A, 2017; RABELOSILVA ER, et., 2018).

A insuficiência cardíaca é uma patologia que afeta aproximadamente $2 \%$ da população mundial, ou seja, mais de 23 milhões de pessoas e 2 milhões de novos casos anualmente. No Brasil, estima-se que 6, 4 milhões de pessoas possuem esta doença, cuja mortalidade aumentou nas últimas três décadas, liderando as causas de morte em 2003. No ano de 2007, foram registradas 293.473 internações, representando $2,59 \%$ dos recursos em saúde, em 2012, 238 mil internações, com ocorrência de 26 mil óbitos e mortalidade de 9,5\% durante a internação (WAJNER A, 2017; NASCIMENTO MNR, et. al., 2019; PAIVA LA, et al., 2019; SILVA WT, et al., 2020).

Em 2016, foi considerada a maior causa de morbimortalidade pelo Sistema Único de Saúde (SUS), responsável por $12.2 \%$ dos gastos financeiros e com aumento de $34 \%$ da mortalidade. Estima-se que até 2025, será responsável por mais da metade das doenças cardiovasculares e mortalidade de $50 \%$ após 5 anos do diagnóstico (WAJNER A, 2017; NASCIMENTO MNR, et. al, 2019; PAIVA LA, et al., 2019; SILVA WT, et al., 2020).

A falta de adesão ao tratamento é a principal causa de internação entre esses pacientes, além do não reconhecimento dos sinais de exacerbação, onde alguns estudos referem $50 \%$ de readmissões hospitalares destes pacientes em até 90 dias, evoluindo com pior prognóstico. No estudo Candesartan in Heart failure: Assessment of Reduction in Mortality and Morbidity (CHARM) realizado por MacDonald MR, et al. (2008), $11 \%$ dos pacientes utilizavam apenas $80 \%$ das medicações prescritas (SOUSA MM, et al., 2017; WEJNER A, 2017; RABELO-SILVA ER, et., 2018; SANTOS EB e BIANCO HT, 2018; ROCHA RC e FIGUEIREDO LF, 2019).

Segundo o Brazilian Registry of Acute Heart Failure (BREATHE), a mortalidade intra-hospitalar é 12,6\%. Dessa forma, tratamento farmacológico deve ser otimizado com betabloqueadores, ácido acetilsalićlico, estatinas e inibidores de enzima de conversão ou bloqueadores de receptores de angiotensina, além da restrição hídrica, de sódio e controle glicêmico (ALBUQUERQUE DC, et al., 2015; SOUSA MM, et al., 2017; WEJNER A, 2017; RABELO-SILVA ER, et., 2018; SANTOS EB e BIANCO HT, 2018; ROCHA RC e FIGUEIREDO LF, 2019). 
A insuficiência cardíaca diastólica (principal tipo relacionado aos diabetes) é demonstrada a muito tempo em diversos trabalhos como o realizado por Yip GW, et al. (1999) que evidenciou 66\% dos pacientes com apresentavam função sistólica normal. O'Connor CM, et al. (2000) relatou que $40 \%$ dos 2.498 pacientes estudados e distribuídos entres as classes II a IV da New York Heart Association (NYHA), apresentavam função sistólica com média de 58\%. Através do Euro Heart Failure Survey, Cleland J (2001), os dados mostraram que em $58 \%$ dos casos estudados, metade apresentava fração da ejeção (FE) ventricular esquerda maior do que $40 \%$ ao ecodopplercardiograma (MARTINS JÚNIOR L, 2017).

Ao nos basearmos nas definições e estatísticas acima, observamos a importância da prevenção e tratamento da miocardiopatia nos diabéticos, sendo fundamental ao médico, e principalmente o cardiologista, realizar o rastreio e tratamento precoce, além de orientar seus pacientes quanto às consequencias do diabetes em geral (SALDARRIAGA-GIRALDO C, et al., 2019; PAVON-ROJAS AJ, et al. 2020; WEBER TR, et al., 2020).

\section{Fisiopatologia do Diabetes no Miocárdio}

A Sociedade Europeia de Cardiologia (ESC) e a Sociedade Européia para Estudos da Diabetes caracterizam a miocardiopatia diabética como uma entidade única, onde a maioria das informações disponíveis sobre sua fisiopatologia provém de modelos experimentais animais. Embora seu diagnóstico precoce seja importante, pois as lesões iniciais podem ser reversíveis, ainda é pouco conhecida pelos médicos em geral, sendo a alteração no relaxamento e aumento da rigidez do ventrículo esquerdo sua característica (RAMIREZ R, et al., 2017; SALDARRIAGA-GIRALDO C, et al., 2019; WEBER TR, et al., 2020).

Diversos são os mecanismos desencadeados pelo diabetes que levam à lesão miocárdica: produtos da glicação avançada, fibrose, inflamação, apoptose, lesões autoimunes, metabolismo dos lipídios, alteração de vias metabólicas, alterações do sistema nervoso autônomo e do sistema ubiquitina-proteossoma (ARAÚJO ASP, 2018; SALDARRIAGA-GIRALDO C, et al., 2019).

Os principais tipos de diabetes envolvidos na miocardiopatia são o DM1 e DM2. O primeiro, de origem autoimune, relaciona-se ao Complexo Principal de Histocompatibilidade (MHC), principalmente os presentes no braço curto do cromossomo 6, sendo os haplótipos HLA-DR4-DQ8 e HLA-DR3-DQ2 os responsáveis por maior risco de desenvolver a doença e de anticorpos anti-célula beta. Mais de 90\% das pessoas com DM1 têm um destes dois haplótipos e cerca de $30 \%$ têm os dois. A presença de células reativas e autoanticorpos está presente anos antes das manifestações clínicas, onde as células mais comuns são linfócitos TCD8, TCD4, células B e macrófagos (COSTA BB e MOREIRA TA, 2021; RAMALHO S e NORTADAS R, 2021).

O DM2 é mais comum, relaciona-se com a resistência à insulina, onde a obesidade é o maior fator de risco, responsável por estados de hiperglicemia e hiperlipidemia com alterações na secreção de insulina pelas células beta. O risco de insuficiência cardíaca nesses pacientes é maior, independente de infarto do miocárdio ou hipertensão arterial prévios. O Framingham Heart Study, evidenciou que $19 \%$ dos doentes avaliados com IC tem DM2 e um aumento de 1\% na hemoglobina glicada aumenta $8 \%$ o risco de IC, independentemente da idade, índice de massa corpórea ou doença arterial coronariana (ROMANCIUC M, 2017; ARAÚJO ASP, 2018; KUL S, et al., 2018).

As alterações glicêmicas levam a desajustes moleculares como presença dos produtos de glicação avançada, definidos como lipídios ou proteínas submetidos à desglicosilação devido à hiperglicemia induzindo fibrose ao impedir degradação do colágeno na matriz extracelular cardíaca, além de estimularem a ativação do fator nuclear $\mathrm{B}$ que aumenta o número de cadeias pesadas de miosina, alterando a recaptação do cálcio. O IGFBP7 (fator de crescimento semelhante à insulina ligado à proteína 7) é um biomarcador identificado na disfunção diastólica associado com fibrose miocárdica e remodelação vascular, sendo clicamente importante sua detecção precoce (ARAÚJO APS, 2018; COELHO RCG, 2018; VERA NC, et al., 2018; SALDARRIAGAGIRALDO C, et al., 2019; PAVON-ROJAS AJ, et al. 2020).

O processo inflamatório crônico leva à disfunção endotelial devido à redução da atividade da enzima óxido nítrico sintase e do óxido nítrico, assim como da prostaciclina, levando à redução da vasodilatação dependente do endotélio, soma-se a isso aumento de endotelina-1 e angiotensina-II, que geram 
vasoconstrição e remodelamento microvascular. O sistema ubiquitina-proteossoma degrada as proteínas danificadas e oxidadas, estando alterado no coração de diabéticos e desencadeia remodelação patológica (ARAÚJO APS, 2018; COELHO RCG, 2018; VERA NC, et al., 2018; SALDARRIAGA-GIRALDO C, et al., 2019).

Fatores pró-trombóticos e moléculas de adesão leucocitária geram agregação plaquetária e inflamação local. O fator de nacrose tumoral alfa somado à interleucina 6 podem exercer efeito inotrópico negativo, induzir fibrose, hipertrofia e morte celular nos cardiomiócitos. No diabético, a troca do metabolismo da glicose pelo uso dos lipídios diminui a eficiência cardíaca e gera desacoplamento mitocondrial, que causa depleção de energia e induz estresse oxidativo (COELHO RCG, 2018; VERA NC, et al., 2018; SALDARRIAGA-GIRALDO C, et al., 2019).

A alteração do sistema nervoso também apresenta importância na fisiopatologia. A neuropatia diabética acarreta deformações relacionadas à estrutura e funcionalidade das fibras nervosas e está diretamente relacionada ao alto índice de mortalidade nos seus portadores. No coração é representada pela neuropatia autonômica cardiovascular (NAC) com prevalência maior que $50 \%$ nos doentes crônicos e manifesta-se como diminuição da variabilidade da frequência cardíaca devido à desnervação parassimpática (PAVON-ROJAS AJ, et al. 2020; SILVA RR, et al., 2021).

Diabéticos com características de disfunção autonômica cardíaca, como taquicardia inexplicada, hipotensão ortostática e baixa tolerância ao exercício devem ser avaliados e sua triagem deve ser realizada no diagnóstico de DM2 e 5 anos após o diagnóstico de DM1. A ativação do sistema simpático aumenta a expressão beta1-adrenérgica que promove hipertrofia, fibrose, apoptose e redução da função contrátil do cardiomiócito, em contrapartida, o sistema parassimpático fica retraído, com alteração dos receptores muscarínicos e diminuição da acetilcolinesterase (PAVON-ROJAS AJ, et al. 2020; SILVA RR, et al., 2021).

\section{Manifestações Clínicas, Diagnóstico e Tratamento}

A Miocardiopatia Diabética, por ser ICFEP, é definida pelo Colégio Americano de Cardiologia (ACC) e pela ESC como a presença de sinais e sintomas de insuficiência cardíaca nos pacientes com Fração de Ejeção (FE) do ventrículo esquerdo igual ou superior a 50\%. A SBC define como FE maior ou igual a 50\%, com índice de volume diastólico final do Ventrículo Esquerdo (VE) menor do que $97 \mathrm{~mL} / \mathrm{m}^{2}$, além dos seguintes dados hemodinâmicos: pressão capilar pulmonar maior do que $12 \mathrm{mmHg}$ e pressão diastólica final do VE maior do que 16 mmHg (GONÇALVES MJG, 2017; MARTINS JÚNIOR L, 2017).

A insuficiência cardíaca é uma síndrome complexa, progressiva, com características peculiares, sendo resultado final de diversas patologias cardiovasculares que irão alterar a qualidade de vida dos pacientes, além de elevar morbimortalidade, caracterizada pela incapacidade do coração de bombear adequadamente o sangue para os demais órgãos e tecidos, ou seja, temos um baixo débito cardíaco e/ou elevadas pressões de enchimento ventricular (POFFO MR, et al., 2017; SBC, 2018; ROCHA RC e FIGUEIREDO LF, 2019).

É composta por sinais e sintomas como dispnéia, edema maleolar, fadiga, tosse, pressão venosa jugular elevada, crepitações pulmonares e alterações da estrutura pulmonar. Pode ser determinada conforme fração de ejeção (preservada, intermediária e reduzida), perfil hemodinâmico, gravidade dos sintomas (classificação funcional da New York Heart Association - NYHA), tempo e progressão da doença (diferentes estágios) (MARQUES MCM, et al., 2016; SBC, 2018).

Conforme o ACC e American Heart Association (AHA) a progressão da doença leva à alterações estruturais cardíacas, dessa forma, apresentando fases sintomáticas e assintomáticas, com condutas terapêuticas peculiares, classificando o paciente em estágios de A a D. A New York Heart Association (NYHA) basea-se na qualidade de vida e capacidade do paciente de executar suas atividades diárias ou ser totalmente incapaz de fazê-las, apresentando quatro classes funcionais I a IV conforme o grau de esforço necessário para gerar algum sintoma (FREITAS EMM, 2018).

O paciente é classificado quanto ao seu perfil clínico-hemodinâmico da seguinte forma: perfil $A$, definido como portador de insuficiência cardíaca, porém compensado clinicamente e assintomático; perfil B (quente e úmido), congesto e bem perfundido; perfil $C$ (frio e úmido), congesto e mal perfundido; perfil $L$ (frio e seco), não congesto e mal perfundido (SBC, 2018; ROCHA RC e FIGUEIREDO LF, 2019). 
Arritmias podem descompensar portadores de insuficiência cardíaca, com destaque para fibrilação atrial que, independente da função sistólica, diminui o inotropismo miocárdico, o débito cardíaco pela perda da sístole atrial, aumento da demanda de oxigênio, redução do aporte sanguíneo para artérias coronárias, além da incapacidade de relaxamento do miocárdio, sendo a principal arritmia responsável por eventos tromboembólicos de origem cardíaca (SBC, 2018; PAIVA LA, et al., 2019).

O diagnóstico é de "exclusão" porque os sinais e sintomas são inespecíficos e não existe uma metodologia padrão-ouro ou biomarcador único para efetuar o diagnóstico correto. Dessa forma, a ESC preconiza que o diagnóstico requer a presença dos seguintes critérios, além dos mencionados acima: concentrações plasmáticas elevadas de peptídeos natriuréticos e evidência objetiva de alterações funcionais e estruturais cardíacas, geralmente diagnosticadas pelo ecodopplercardiograma (GONÇALVES MJG, 2017; FERNANDES-SILVA MM e BARBERATO SH, 2020).

A Associação de Insuficiência Cardíaca da ESC criou o algoritmo HFA-PEFF no qual, escore maior ou igual a 5, o diagnóstico de ICFEP é confirmado. Se for intermediário (2 a 4 pontos) indiaca-se teste de estresse ou medidas invasivas. Se o paciente preencher um ponto ou menos, o diagnóstico fica afastado (FERNANDES-SILVA MM e BARBERATO SH, 2020).

O ecodopplercardiograma é um método barato e fundamental na elucidação diagnóstica, onde o doppler tecidual mede as velocidades do tecido miocárdico durante o ciclo cardíaco, dessa forma, estimando a função global sistólica e diastólica do coração. Existem três tipos de disfunção diastólica: grau I (relaxamento ventricular diminuído sem aumento das pressões de enchimento), grau II (relaxamento diminuído com aumento das pressões de enchimento) e grau III (pressões de enchimento muito elevadas com padrão restritivo do fluxo mitral). Através deste exame, pacientes diabéticos tipo I, podem apresentar alterações mesmo no diagnóstico recente, inferindo cardiomiopatia diabética incipiente. A ressonância cardíaca também é útil na detecção de esteatose miocárdica e disfunção diastólica (VERA NC, et al., 2018; FERNANDESSILVA MM e BARBERATO SH, 2020; WEBER TR, et al., 2020).

É notório que os portadores de ICFEP são obesos em sua maioria e que atividade física está associada à redução de doenças cardiovasculares e mortalidade em diabéticos (VERA NC, et al., 2018). O estudo de reabilitação cardíaca realizado por Moraes RS, et al. (2005) apud Firmino SM (2021) mostrou melhora na capacidade funcional mesmo com atividades de pequena duração, aumento no tempo de exercício e na distância percorrida no tempo de caminhada de seis minutos.

Segundo Shah SH, et al. (2016) apud Gonçalves MJG (2017) vinte semanas de dieta com restrição calórica foram seguras em doentes idosos e obesos com ICFEP, demonstrando melhora nos sintomas, consumo de oxigênio e qualidade de vida, sendo o aumento do consumo de oxigênio relacionado à diminuição do índice de massa corporal e dos marcadores inflamatórios. O ajuste dietético diminui as anomalias metabólicas desencadeadas pelo diabetes e a resistência sistêmica à insulina, aumentando sensibilidade à glicose mediada pelo transporte de insulina e sinalização pós-receptor de insulina. O controle glicêmico retarda a miocardiopatia, reduz esteatose miocárdica e melhora parâmetros em ressonância cardíaca (VERA NC, et al., 2018).

Pelo fato da disfunção microvascular coronária ser o cerne da miocardiopatia diabética e ser desencadeada pela hiperglicemia, devemos incentivar todos os pacientes a manter níveis glicêmicos baixos, retardando o avanço da doença. No United Kingdon Prospective Diabetes Study (UKPDS) realizado por King $P$, et al. (1999) com diabéticos tipo II, o controle rigoroso da glicemia melhorou a disfunção ventricular, porém não foi corroborado por outros como ACCORD, ADVANCE e o VADT, que não demonstraram redução do risco cardiovascular com controle glicêmico (ARAÚJO ASP, 2018).

Do ponto de vista medicamentoso, as drogas consagradas na insuficiência cardíaca com fração de ejeção reduzida (ICFER ou IC sistólica), não apresentaram bons resultados no contexto de morbi-mortalidade na ICFEP, ou seja, os Inibidores da Enzima Conversora de Angiotensina (IECA), Bloqueadores dos Receptores de Angiotensina (BRA), beta-bloqueadores cardioseletivos, antagonistas dos receptores de aldosterona e a associação valsartana/sacubitril (BRA/inibidor da neprilisina). Devemos avaliar cada paciente em corrigir fatores de risco: hipertensão arterial descontrolada, frequência cardíaca elevada, controle de volemia e sinais de isquemia miocárdica (GONÇALVES MJG, 2017; MARTINS JÚNIOR L, 2017; ARAÚJO ASP, 2018). 
Os benefícios presentes nas principais drogas são: diuréticos diminuem o enchimento do ventrículo esquerdo, melhorando sintomas de congestão com redução da pressão na artéria pulmonar e melhora o enchimento do ventrículo direito; ácido acetilsalicílico reduz agregação plaquetária e estatinas diminuem disfunção endotelial e restabelecem a biodisponibilidade do óxido nítrico, sendo indicados a todos os diabéticos com angina microvascular, além dos beta-bloqueadores, pois reduzem as necessidades metabólicas do miocárdio e aumentam a perfusão diastólica ao diminuir a frequência cardíaca. Os antagonistas dos canais de cálcio foram benéficos em modelos animais (GONÇALVES MJG, 2017; COELHO RCG, 2018; VERA NC, et al., 2018).

Os inibidores do cotransportador sódio-glicose 2 (SGLT2), responsável por causar glicosúria em diabéticos tipo II, reduzem os níveis de hemoglobina glicosilada através do controle da gordura visceral, pressão e rigidez arteriais, reduzem peso, albuminúria, hiperinsulinemia e estresse oxidativo, além de diminuir as hospitalizações nesse grupo (VERA NC, et al., 2018; SALDARRIAGA-GIRALDO C, et al., 2019).

\section{CONSIDERAÇÕES FINAIS}

A miocardiopatia diabética é uma doença grave que piora no decorrer de sua evolução, aumentando morbimortalidade e reduzindo qualidade de vida. Dessa forma, precisa ser investigada desde as primeiras consultas cardiológicas do paciente com objetivo de reduzirmos os fatores de risco, introduzir tratamento medicamentoso adequado para cada paciente e conscientizarmos os pacientes sobre estilos de vida mais saudáveis. É uma doença que ainda precisa de mais estudos prospectivos para definição de tratamentos mais específicos, porém com chances muito promissoras nos próximos anos.

\section{REFERÊNCIAS}

1. ALBUQUERQUE DC, et al. Brazilian Registry of Heart Failure - Clinical Aspects, Care Quality and Hospitalization Outcomes. Arquivos Brasileiros de Cardiologia, 2015; 104(6): 433-442.

2. ARAÚJO ASP. Cardiomiopatia Diabética. Dissertação (Mestrado Integrado em Medicina) - Faculdade de Medicina. Universidade do Porto, Porto, 2018; $54 \mathrm{p}$.

3. CASTANHOLA ME, PICCININ A. Fisiopatologia da diabetes e mecanismo de ação da insulina revisão de literatura. $9^{\circ}$ Jornada Científica e Tecnológica da Fatec de Botucatu. São Paulo: 2020.

4. COELHO RCG. Disfunção microvascular coronária em doentes diabéticos. Dissertação (Mestrado Integrado em Medicina) - Clínica Universitária de Cardiologia. Universidade do Porto, Porto, 2018; 49 p.

5. COSTA BB, MOREIRA TA. Principais aspectos fisiopatológicos e clínicos presentes no Diabetes mellitus tipo I (autoimune). Research, Society and Development, 2021; 10(14): 1-7.

6. FERNANDES-SILVA MM, BARBERATO SH. O que o cardiologista espera do ecocardiograma na insuficiência cardíaca com fração de ejeção preservada? ABC, Imagem cardiovascular, 2020; 33(1): 1-2.

7. FIRMINO SM. Associação de pré-diabetes com marcadores precoces de lesão cardiovascular. Tese (Doutorado em Biotecnologia) - Centro de Ciências Exatas e de Tecnologia. Universidade Federal de São Carlos, São Carlos, 2021; $116 \mathrm{p}$.

8. FREITAS EMM. Adesão ao tratamento medicamentoso em insuficiência cardíaca. Dissertação (Mestrado em Ciências da Saúde) - Programa de Pós-Graduação em Ciências da Saúde. Universidade Federal de Goiás, Goiânia, 2018 ; 95.

9. GONÇALVES MJGS. Tratamento da Insuficiência Cardíaca com Fração de Ejeção Preservada. Dissertação (Mestrado Integrado em Medicina) - Faculdade de Medicina. Universidade do Porto, Porto, 2017; 55 p.

10. KING P, et al. The UK Prospective Diabetes Study (UKPDS): clinical and therapeutic implications for type 2 diabetes. British Journal of Clinical Pharmacology, 1999; 48(5): 643-648.

11. KUL S, et al. Onda pressistólica associada à disfunção ventricular esquerda subclínica e avaliada com base no indice de performance miocárdica em pacientes com diabetes mellitus tipo 2. Arquivos Brasileiros de Cardiologia, 2019; 113(2): 207-215.

12. LUNDBAEK K. Is there a diabetic cardiopathy? Pathogenetische faktoren des myokardinfarkts. Stuttgart: Schattauer; 1969: 63-71.

13. MACDONALD MR, et al. Impact of diabetes on outcomes in patients with low and preserved ejection fraction heart failure: an analysis of the Candesartan in Heart failure: Assessment of Reduction in Mortality and morbidity (CHARM) programme. European Heart Journal, 2008; 29: 1377-1385.

14. MARTINS JÚNIOR L. Insuficiência cardíaca diastólica. Revista da Faculdade de Ciências Médicas de Sorocaba. 2017; 19(2): 92-93. 
15. MARQUES MCM, et al. Autocuidado no doente com insuficiência cardíaca. Revista lbero-America de Saúde e Envelhecimento, 2016; 2(1): 439-452.

16. MORAES RS, et al. Diretriz de reabilitação cardíaca. Arquivos Brasileiros de Cardiologia, 2005: 84(5): $431-440$.

17. NASCIMENTO MNR, et al. Aspectos da assistência de enfermagem para pessoa com insuficiência cardíaca. Revista de Enfermagem e Atenção à Saúde, 2019; 8(2): 123-134.

18. O'CONNOR CM, et al. Clinical characteristics and long-term out comes of patients with heart failure and preserved systolic function. American Journal of Cardiology, 2000; 86: 863-867.

19. PAIVA LA, et al. Uma abordagem sobre as causas de descompensação em insuficiência cardíaca crônica: relato de caso. V Seminário Científico do UNIFACIG - IV Jornada de Iniciação Científica do UNIFACIG, 2019: 1-5.

20. PAVÓN-ROJAS AJ, et al. Un acercamiento a las bases moleculares de la miocardiopatía diabética. Scalpelo, 2020; 1(2): 48-55.

21. POFFO MR, et al. Perfil dos Pacientes Internados por Insuficiência Cardíaca em Hospital Terciário. International Journal of Cardiovascular Sciences, 2017; 30(3): 189-198.

22. RABELO-SILVA ER, et al. Fatores precipitantes de descompensação da insuficiência cardíaca relacionados a adesão ao tratamento: estudo multicêntrico-EMBRACE. Revista Gaúcha de Enfermagem, 2018; 39: 1-6.

23. RAMALHO S, NORTADAS R. Anticorpos na diabetes mellitus tipo 1. Revista Portuguesa de Diabetes, 2021; 16(2): 73-79.

24. RAMIREZ R, et al. Miocardiopatía diabética: un punto de vista retrospectivo. Revista Colombiana de Cardiología, 2018; 25(1): 51-54.

25. ROCHA RC, FIGUEIREDO LF. O perfil do paciente internado com insuficiência cardíaca no hospital das clínicas de Teresópolis. Revista da Faculdade de Medicina de Teresópolis, 2019; 3(1): 69-78.

26. ROMANCIUC M. Diabetes Mellitus Tipo 2 como Doença Inflamatória: anatomia, fisiopatologia e terapêutica. Dissertação (Mestrado em Ciências Farmacêuticas) - Faculdade de Ciências e Tecnologia. Universidade do Algarve, Faro, 2017; 75 p.

27. RUBLER S, et al. New type of cardiomyopathy associated with diabetic glomerulosclerosis. American Journal of Cardiology, 1972; 30: 595-602.

28. SALDARRIAGA-GIRALDO C, et al. De la diabetes a la insuficiencia cardiaca ¿Existe la miocardiopatía diabética? Revista Colombiana de Cardiología, 2020; 27(S2): 12-16.

29. SANTOS EB, BIANCO HT. Atualizações em doença cardíaca isquêmica aguda e crônica. Revista da Sociedade Brasileira de Clínica Médica, 2018; 16(1): 52-58.

30. SHAH SJ, et al. Phenotype-specific treatment of heart failure with preserved ejection fraction. Circulation, 2016; 134(1): 73-90.

31. SILVA RR, et al. Avaliação dos fatores de risco e diagnóstico para neuropatia autonômica cardíaca em pessoas diabéticas. Global Academic Nursing Journal, 2021; 2(Spe.3): e164: 1-7.

32. SILVA TRW. Correlação entre neuropatia autonômica cardiovascular e cardiomiopatia diabética no diabetes tipo 1. Dissertação (Mestrado em Medicina) - Centro de Ciências da Saúde. Universidade Federal de Santa Catarina, Florianópolis, 2019; 72 p.

33. SILVA WT, et al. Características clínicas e comorbidades associadas à mortalidade por insuficiência cardíaca em um hospital de alta complexidade na Região Amazônica do Brasil. Revista Pan-Amazônica de Saúde, 2020; 11: 3-8.

34. SOCIEDADE BRASILEIRA DE CARDIOLOGIA (SBC). Diretriz Brasileira de Insuficiência Cardíaca Crônica e Aguda. Arquivos Brasileiros de Cardiologia, 2018; 111(3): 436-539.

35. SOUSA MM, et al. Associação das condições sociais e clínicas à qualidade de vida de pacientes com insuficiência cardíaca. Revista Gaúcha de Enfermagem, 2017; 38(2): 1-8.

36. VERA NC, et al. Miocardiopatía Diabética, lo que hoy conocemos. Revista Cubana de Cardiología y Cirugía Cardiovascular, 2018; 24(1): 1-25.

37. WAJNER A. Causas e preditores de mortalidade em pacientes que Internam com ou por insuficiência cardíaca em Hospital terciário no Brasil. Tese (Doutorado em Cardiologia e Ciências Cardiovasculares) - Faculdade de Medicina. Universidade Federal do Rio Grande do Sul, Porto Alegre, 2017; 60 p.

38. WEBER TR, et al. Avaliação ecocardiográfica na diabetes mellitus tipo 1. Revista Portuguesa de Cardiologia, 2021; 40(10): 757-765.

39. YIP GW, et al. Comparisonand systolic and diastolic heart failure in Chinese living in Hong Kong. American Journal of Cardiology, 1999; 84: 563-567. 